\title{
Partial Solvers for Parity Games: Effective Polynomial-Time Composition
}

\author{
Patrick Ah-Fat and Michael Huth \\ Imperial College London \\ London, UK \\ Department of Computing \\ \{patrick-ah-fat14, m.huth\}@imperial.ac.uk
}

\begin{abstract}
Partial methods play an important role in formal methods and beyond. Recently such methods were developed for parity games, where polynomial-time partial solvers decide the winners of a subset of nodes. We investigate here how effective polynomial-time partial solvers can be by studying interactions of partial solvers based on generic composition patterns that preserve polynomial-time computability. We show that use of such composition patterns discovers new partial solvers - including those that merge node sets that have the same but unknown winner - by studying games that composed partial solvers can neither solve nor simplify. We experimentally validate that this data-driven approach to refinement leads to polynomial-time partial solvers that can solve all standard benchmarks of structured games. For one of these polynomial-time partial solvers not even a sole random game from a few billion random games of varying configuration was found that it won't solve completely.
\end{abstract}

\section{Introduction}

Parity games are two-player games on directed graphs that are determined [18, 4, 21. Parity games have several applications, including as back-ends in formal methods. The exact computational complexity for finite parity games has been an open problem for over 20 years: deciding which player wins a node in a parity game is in UP^coUP [16] and the fastest known algorithms run in sub-exponential time in the size of games, see e.g. [15, 19]. Some types of parity games have polynomial-time solutions. Bounding the index of games - i.e. the largest color of a game - by a fixed number, Zielonka's algorithm based on the whole-set rule [8] becomes polynomial time. Or we may bound a descriptive complexity measure: parity games with bounded DAG-width [2], tree-width [2, 5] or entanglement [3] can be solved in polynomial time.

Algorithms that solve parity games do so using specific mechanisms, for example strategy improvement [20] or progress measures [14]. But it seems not feasible to let such mechanisms interact in iterative computations, even though this might speed up solving time. The difficulty is that such mechanisms operate over very different views of games and their complexity; for example, how might one use a strategy-improvement step (which updates one player's strategy) to increase a progress measure (an element in a specific complete lattice)?

Partial solvers [10, 11] have been proposed as algorithms that can solve parts of a parity game but not necessarily all of such a game. Such algorithms are designed to run in polynomial time, and this is relatively easy to obtain. The harder part is to understand which parity games are solved completely by a given partial solver. Partial solvers are related to known static analyses such as priority propagation (see e.g. [7]), that may decrease colours of nodes. Extant work

D. Cantone and G. Delzanno (Eds.): Seventh Symposium on Games, Automata, Logics and Formal Verification (GandALF'16)

EPTCS 226, 2016, pp. 1-15 doi 10.4204/EPTCS.226.1 (c) P. Ah-Fat \& M. Huth

This work is licensed under the Creative Commons Attribution License. 
has shown the feasibility of using partial solvers [10, 11, yet they don't completely solve some benchmarks of structured games and they don't solve many randomly generated games. In this paper, we ask whether partial solvers can improve their effectiveness through interaction.

Addressing this question seems feasible as all these methods share a common view of the complexity of a finite game - say the number of nodes plus the number of edges plus the sum of all colours of all nodes. This common view allows us to think of static analyses, let us mention color reductions based on abstract Rabin index computations [9], as partial solvers as well and to then compose partial solvers to improve their effectiveness. This discussion leads us to consider whether there are simple, generic, yet effective composition patterns for partial solvers that preserve polynomial-time computability, allow us to manually discover new partial solvers, and that can solve all standard benchmarks of structured games and only very rarely do not completely solve a randomly generated game. The main contribution of this paper is to provide such an approach and experimental evidence that such aims are realizable.

Outline of paper: We review background in Section 2, develop our composition approach for partial solvers in Section 3 , and show how its use leads to data-driven refinement of partial solvers in Section 4. In Section 5, we report our experimental and validation work for our approach and its newly discovered partial solvers. Related work is discussed in Section 6, further insights are discussed in Section 7, and Section 8 concludes the paper. We refer to [1] for proofs, a formal presentation of our approach, and further experimental details not provided in this paper.

\section{Background}

We define key concepts of parity games, review some partial solvers and static analyses for such games, and fix technical notation used in this paper. We write $\mathbb{N}$ for the set $\{0,1, \ldots\}$ of natural numbers. A parity game $G$ is a tuple $\left(V, V_{0}, V_{1}, E, c\right)$, where $V$ is a set of nodes partitioned into possibly empty node sets $V_{0}$ and $V_{1}$, with an edge relation $E \subseteq V \times V$ that contains no dead-ends (i.e. for all $v$ in $V$ there is a $w$ in $V$ with $(v, w)$ in $E$ ), and a colouring function $c: V \rightarrow \mathbb{N}$. Throughout, we write $p$ for one of 0 or 1 and $1-p$ for the other player. Nodes in $V_{0}$ are owned by player 0 , nodes in $V_{1}$ are owned by player 1 . We write owner $(v)$ to denote the $p$ for which $v$ is in $V_{p}$. In figures, $c(v)$ is written within nodes $v$, nodes in $V_{0}$ are depicted as circles and nodes in $V_{1}$ as squares. For a relation $\rho \subseteq A \times B$ and $X \subseteq A$ we write $X \bullet \rho$ for set $\{b \in B \mid \exists a \in X:(a, b) \in \rho\}$, whereas $\rho \bullet Y$ denotes set $\{a \in A \mid \exists b \in Y:(a, b) \in \rho\}$ for $Y \subseteq B$; we will abuse this notation for singleton $X$ and $Y$ as in $v \bullet E$ or $E \bullet v$ in a parity game. Below we write $\mathrm{C}(G)$ for the set of colours in game $G$, i.e.

$$
\mathrm{C}(G)=\{c(v) \mid v \in V\}
$$

We write $x \% 2$ for $x$ modulo 2 for an integer $x$. For each $p$ in $\{0,1\}$, the preference ordering $\preceq_{p}$ on $\mathrm{C}(G)$ is given by $c_{1} \preceq_{p} c_{2}$ iff $\left(c_{1} \% 2=p\right.$ and $\left.c_{2} \% 2=1-p\right)$ or $\left(c_{1}\right.$ and $c_{2}$ have parity $p$ and $\left.c_{1} \leq c_{2}\right)$ or $\left(c_{1}\right.$ and $c_{2}$ have parity $1-p$ and $\left.c_{2} \leq c_{1}\right)$.

A play from some node $v_{0}$ results in an infinite play $\pi=v_{0} v_{1} \ldots$ in $(V, E)$ where the player who owns $v_{i}$ chooses the successor $v_{i+1}$ such that $\left(v_{i}, v_{i+1}\right)$ is in $E$. Let $\operatorname{lnf}(\pi)$ be the set of colours that occur in $\pi$ infinitely often:

$$
\operatorname{lnf}(\pi)=\left\{k \in \mathbb{N} \mid \forall j \in \mathbb{N}: \exists i \in \mathbb{N}: i>j \text { and } k=c\left(v_{i}\right)\right\}
$$


Player 0 wins play $\pi$ iff $\min \operatorname{lnf}(\pi)$ is even; otherwise player 1 wins play $\pi$. A strategy for player $p$ is a total function $\sigma_{p}: V^{*} \cdot V_{p} \rightarrow V$ where the pair $\left(v, \sigma_{p}(w \cdot v)\right)$ is in $E$ for all $v$ in $V_{p}$ and $w$ in $V^{*}$. A play $\pi$ conforms with $\sigma_{p}$ if for every finite prefix $v_{0} \ldots v_{i}$ of $\pi$ with $v_{i}$ in $V_{p}$ we have $v_{i+1}=\sigma_{p}\left(v_{0} \ldots v_{i}\right)$.

Parity games are determined [18, 4, 21]: (i) node set $V$ is the disjoint union of two, possibly empty, sets $\operatorname{Win}_{0}[G]$ and $\operatorname{Win}_{1}[G]$, the winning regions of players 0 and 1 (respectively) in $G$; and (ii) there are (memoryless) strategies $\sigma_{0}$ and $\sigma_{1}$ such that all plays beginning in $\mathrm{Win}_{0}[G]$ and conforming with $\sigma_{0}$ are won by player 0 , and all plays beginning in $\operatorname{Win}_{1}[G]$ and conforming with $\sigma_{1}$ are won by player 1 . By abuse of language, $\emptyset$ is also a parity game with no nodes.

We define the rank of parity game $G$ as

$$
\mathrm{r}(G)=|V|+|E|+\sum_{v \in V} c(v)
$$

We write $\operatorname{Attr}_{p}[G, X]$ for the attractor of node set $X$ for player $p$, which computes the alternating reachability of $X$ for that player in the game graph of $G$ (see e.g. Definition 1 in [10]). It is well known that $\operatorname{Attr}_{p}[G, X]$ is contained in the winning region $\operatorname{Win}_{p}[G]$ whenever $X \subseteq \operatorname{Win}_{p}[G]$. The color of a finite path or cycle $P$ in the directed graph $(V, E)$ is defined to be $\min \{c(v) \mid v$ is on $P\}$. A subset $C \subseteq V$ of a directed graph is called a (maximal) strongly connected component, denoted by SCC, if for all $v, w$ in $C$ there is a path in $(V, E)$ from $v$ to $w$; and if there is no strict superset of $C$ in $(V, E)$ with that property.

Example 1 For parity game $G$ on the right in Figure 2, we have $\operatorname{Win}_{1}[G]=\emptyset$ and $\operatorname{Win}_{0}[G]=V$. The (memoryless) strategy $\sigma_{0}$ with $\sigma_{0}\left(v_{4}\right)=v_{19}$ is a winning strategy for player 0 on $\mathbf{W i n}_{0}[G]$.

We present partial solvers and static analyses for parity games, some of them already in a form suitable for the composition patterns developed in this paper. All these partial solvers and static analyses preserve the winning regions of the (remaining) game, and can be computed in polynomial time in the size of their input games [10, 12, 9]. Static color compression $s c c$ is agnostic to the game graph and makes $\mathrm{C}(G)$ convex in $\mathbb{N}$, e.g. $\mathrm{C}(G)=\{0,2,3,6,7\}$ becomes $\{0,1,2,3\}$ where nodes coloured with 2 now have color 0 , nodes coloured 3 now have color 1 and so forth. Priority propagation $p p$ is informed by the game graph. At node $v$, let $p(v)$ denote $\min (\max c(v \bullet E), \max c(E \bullet v))$ where $c(Y)=\{c(y) \mid y \in Y\}$; if there is a node $v$ with $p(v)<c(v)$, one such node is selected by $p p$ and the color at $v$ is changed to $p(v)$; otherwise $p p$ has no effect.

The monotone attractor for a node set $X$ of color $d$ in $\mathrm{C}(G)$ [10] is defined as follows: it is the greatest set of nodes $Y_{X}$ in $G$ from which player $d \% 2$ can force to reach nodes in $X$ whilst only encountering nodes of color $\geq d$ en route. A node set $X$ is a fatal attractor [10] if it is contained in its monotone attractor $Y_{X}$, and then all nodes in $X$ are won by player $d \% 2$ in parity game $G$ [10]. We write $f a$ for the static analysis that returns a fatal attractor (say by exploring colours in descending order) if $G$ has one, and returns nothing otherwise. Another static analysis ari is based on the abstract Rabin index of parity games [9]: for node $v$ with $c(v)>1$, let $c_{v}^{\prime}$ be the maximal color of all cycles that go through node $v$ in $G$; if there is a node $v$ with $c_{v}^{\prime}<c(v)$, then ari chooses one such node and changes the color at $v$ to $c_{v}^{\prime}$; otherwise ari has no effect on $G$. Finally, let $g f a$ be a more general form of partial solver $f a$, based on the partial solver in [12. For $g f a$, all nodes in node set $X$ have color parity $p$ (not necessarily the same color), and $Y_{X}$ is the greatest set of nodes from which player $p$ can ensure that $X$ is reached such that the minimal color encountered en route has parity $p[12$. Partial solver $g f a$ returns a set $X$ contained in the corresponding $Y_{X}$, if there is such a pair $\left(X, Y_{X}\right)$, and returns nothing otherwise. 


\section{Composition of partial solvers}

We now present our approach to expressing and composing partial solvers with ease. Fundamental to this is the notion of a state $s$ which has form

$$
\left(W_{0}, W_{1}, \rho, G^{\prime}, G\right)
$$

and models an intermediate state of computation within an implicit composition context. Below, we write $s . G$ and so forth to refer to such components of state $s$, write $s . V$, s.E etc. for the components of game $s . G$, and similarly for game $s . G^{\prime}$. We may elide prefix " $s$." if state $s$ is clear from context. The original input game is $s . G$ and parity game $s . G^{\prime}$ is the continuation game that still needs to be solved; node sets $s . W_{p}$ for $p$ in $\{0,1\}$ model those nodes in $s . G$ for which the winner is already decided as player $p$; for $v$ in $V^{\prime}$, node set $v \bullet \rho$ for $\rho \subseteq V^{\prime} \times V$ represents those nodes in $V$ that have the same (not yet known) winner in $s . G$ as $v$ has in $s^{\prime} . G^{\prime}$; and the winning regions $\operatorname{Win}_{p}[s . G]$ of $s . G$ are the union of $s . W_{p}$ and the image of the winning region $\operatorname{Win}_{p}\left[s . G^{\prime}\right]$ under relation $\rho$. A state models configurations of partial solver computations, where

$$
\left(\emptyset, \emptyset, \Delta_{V_{G}}, G, G\right)
$$

is a natural initial configuration with $\Delta_{V_{G}}=\left\{(v, v) \mid v \in V_{G}\right\}$, and the more general configurations model composition contexts.

We write $\Sigma$ for the set of all states, let the rank of $s$ be the rank of $s . G^{\prime}$, and define a partial order $\leq$ on states by

$$
s^{\prime} \leq s \text { iff }\left(s=s^{\prime} \text { or } r\left(s^{\prime}\right)<\mathrm{r}(s)\right)
$$

Note that $(\Sigma, \leq)$ satisfies the descending chain condition, where the length of any descending chain starting in $s$ is polynomial in $\mathrm{r}\left(s . G^{\prime}\right)$.

A partial solver is a terminating algorithm $A$ whose semantics $f$ is a state transformer of type $\Sigma \rightarrow \Sigma$ and satisfies, for all $s$ in $\Sigma$, the following: the input game $s . G$ won't change under $f$, $f$ strictly decreases the rank or won't change the state, and $f$ preserves winners of nodes whose winners have already been decided.

Definition 1 Let $\mathcal{P}$ be the set of partial solvers that run in polynomial time in rank of s.G.

By abuse of language, we sometimes refer to functions $f$ as partial solvers but context will determine which algorithms they denote.

Refinement for state transformers $f \leq g$ is defined as $f \leq g$ iff $\forall s \in \Sigma: f(s) \leq g(s)$. Then $f$ refines $g$, and so any partial solver with semantics $f$ refines any partial solver with semantics $g$. We write $\Sigma_{f}$ for the set of residual games of partial solver $f$, which $f$ cannot simplify.

We now formally present the five analyses from Section 2 in this setting: Static color compression $s c c$ maps a state $s=\left(W_{0}, W_{1}, \rho, G^{\prime}, G\right)$ to $s^{\prime}$ which is $s$ except that $s . G^{\prime}$ may change to reflect the compressed, convex color set. Priority propagation $p p$ also may only change $s . G^{\prime}$ such that the color of at most one node in $s . G^{\prime}$ is decreased and all other aspects of $s . G^{\prime}$ remain the same in $s^{\prime}$. For fatal attractor detection $f a$, suppose it detects a fatal attractor $X$ won by player $p$ in $G$. We set $Z=\operatorname{Attr}_{p}\left[s . G^{\prime}, X\right]$. Partial solver $f a$ then transforms state $s$ into (assuming $p=0$ without loss of generality): $s^{\prime}=\left(W_{0} \cup Z \bullet \rho, W_{1}, \rho^{\prime}, G^{\prime} \backslash Z, G\right)$. where $\rho^{\prime}$ is the restriction of $\rho$ from domain $V^{\prime}$ to $V^{\prime} \backslash Z$, and $G^{\prime} \backslash Z$ is parity game $G^{\prime}$ restricted to node set $V^{\prime} \backslash Z$ (which eliminates all incoming and outgoing edges of $Z$ as well). Next, consider static analysis ari. If there is 
no node $v$ in $s . G^{\prime}$ with $c_{v}^{\prime}<c(v)$, then $\operatorname{ari}(s)=s$. Otherwise, some such node is chosen and $s^{\prime}$ equals $s$ except that $s^{\prime} . G^{\prime}$ reduces the color at node $v$ to $c_{v}^{\prime}$ in $s . G^{\prime}$. The behaviour of $g f a$ is the same as for $f a$ above except that the manner in which such a node set $X$ is computed differs [12], e.g. colours of nodes in $X$ may vary. We summarise the above discussion:

Lemma 1 The partial solvers scc, pp, fa, ari, and gfa have semantics $\Sigma \rightarrow \Sigma$ and are in $\mathcal{P}$.

We are interested in sequential iterations of partial solvers that revert control to the first solver in the sequence as soon as state rank decreases: Let $f_{1}, \ldots, f_{k}$ be elements of $\mathcal{P}$ with $k \geq 1$. Let, for each $s$ in $\Sigma$, set $M_{s}$ be $\left\{i \mid 1 \leq i \leq k, \mathrm{r}\left(f_{i}(s)\right)<\mathrm{r}(s)\right\}$. Then while $\left(f_{1}, \ldots, f_{k}\right)(s)$ is defined as $s$ if $M_{s}=\emptyset$ and as while $\left(f_{1}, \ldots, f_{k}\right)\left(f_{j_{m}}(s)\right)$ otherwise where $j_{m}=\min \left(M_{s}\right)$. It is not hard to show that this defines a family of operators on $\mathcal{P}$ :

Lemma 2 For $k \geq 1$, operator $\lambda\left(f_{1}, \ldots, f_{k}\right)$ while $\left(f_{1}, \ldots, f_{k}\right)$ has type $\mathcal{P}^{k} \rightarrow \mathcal{P}$.

For a partial solver $g=$ while $\left(f_{1}, \ldots, f_{k}\right)$, we have $\Sigma_{g}=\bigcap_{i=1}^{k} \Sigma_{f_{i}}$. In particular, $\Sigma_{g}$ is invariant under permuting the order of the $f_{i}$ in $g$. Operator while $(\cdot)$ supports our data-driven approach to refinement as follows: given $g_{0}=$ while $\left(f_{1}, \ldots, f_{k}\right)$ we study games $s . G^{\prime}$ with while $\left(f_{1}, \ldots, f_{k}\right)(s)=$ $s$ to manually learn a new static analysis $f_{k+1}$ with while $\left(f_{1}, \ldots, f_{k}, f_{k+1}\right)(s) \neq s$, and then similarly consider $g_{1}=$ while $\left(f_{1}, \ldots, f_{k}, f_{k+1}\right)$ on the set of states $\Sigma_{g_{0}}$ for further refinement. These are refinements since while $\left(f_{1}, \ldots, f_{k}\right) \geq$ while $\left(f_{1}, \ldots, f_{k}, f_{k+1}\right)$ for all $k \geq 1$ and all partial solvers $f_{1}, \ldots, f_{k+1}$. The partial solvers in [10, 11, 12] could not completely solve all 1-player games. We show that such completeness is achievable by the interaction of such partial solvers with ari and $s c c^{\prime}$ - a variant of $s c c$ that statically compresses the color set of each SCC in a parity game separately: if $C$ is such a SCC with set of colours $\mathcal{C}$, then $s c c^{\prime}$ makes $\mathcal{C}$ convex in $\mathbb{N}$ and recolours the SCC $C$ accordingly. This also illustrates how we may reason about states in $\Sigma_{g}$ :

Theorem 1 Let $g=$ while $\left(f_{1}, \ldots, f_{k}\right)$ be in $\mathcal{P}$ with $\left\{s c c^{\prime}\right.$, ari,$\left.f a\right\}$ contained in $\left\{f_{1}, \ldots, f_{k}\right\}$. Then there is no $s$ in $\Sigma_{g}$ for which $s . G^{\prime}$ is a 1-player game.

Operator lifted $(f)$ transforms a partial solver $f$ into a second-order version that tests consequences of edge removals on residual games of $f$. For game $G$ with edge relation $E$, this uses derived games: $G_{(v, w)}$ equals $G$ except where $v \bullet E$ is now $\{(v, w)\}$; and $G \backslash(v, w)$ equals $G$ except where $(v, w)$ is removed from $E$. By abuse of notation, we write $s \backslash(v, w)$ for a state that equals state $s$ except that $(s \backslash(v, w)) \cdot G^{\prime}$ equals $G^{\prime} \backslash(v, w)$. That is to say, $G_{(v, w)}$ removes from $E$ all edges $\left(v, w^{\prime}\right)$ with $w \neq w^{\prime}$, whereas $G \backslash(v, w)$ removes from $G$ the edge $(v, w)$. The game $G \backslash(v, w)$ will not introduce deadlocks as it will only be called on nodes $v$ with $|v \bullet E|>1$. We also require notation for initial calling contexts of partial solvers:

$$
\text { call }(f)(G)=\left(f(s) \cdot W_{0}, f(s) \cdot W_{1}\right)
$$

where $s$ equals $\left(\emptyset, \emptyset, \Delta_{V_{G}}, G, G\right)$. Expression call $(f)(G)$ extracts the respective set of nodes that $f$ can decide to be won by each player, when run in an initial configuration for $G$. Operator lifted $(f)$, in Figure 1. tests whether the commitment to edge $(v, w)$ in $G_{(v, w)}$ turns a residual state of $f$ into one that it not residual, and this will allow it to simplify $G$ to either $G_{(v, w)}$ or $G \backslash(v, w)$. Thus, lifted $(\cdot)$ either leaves a state unchanged or removes from $s . G^{\prime}$ at least one edge. We use lifted $(\cdot)$ for defining, for all $f$ in $\mathcal{P}$, function lift $(f): \Sigma \rightarrow \Sigma$ through

$$
\operatorname{lift}(f)=\text { while }(f, \operatorname{lifted}(f))
$$




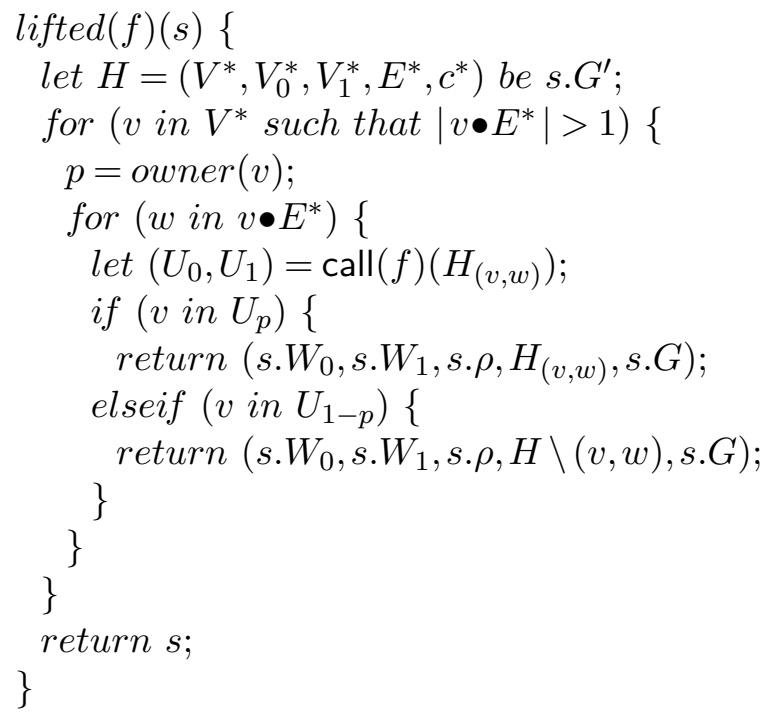

Figure 1: Pseudo-code for function lifted(·) with dependent type $\prod_{f: \mathcal{P}}\left(\Sigma_{f} \rightarrow \Sigma\right)$ : for partial solver $A$ in $\mathcal{P}$ with semantics $f$, it renders a partial solver lifted $(A)$ in $\mathcal{P}$ with semantics lifted $(f): \Sigma_{f} \rightarrow \Sigma$ by testing effects of edge removals on running $A$

Note that $\operatorname{lift}(f)$ now has domain $\Sigma$ as the semantics of while $(\cdot)$ enforces that lifted $(f)$ is only reached with input from $\Sigma_{f}$. Let algorithm $A$ have semantics $f$; we write lifted $(A)$ for the algorithm obtained from the pseudo-code for lifted $(f)$ in Figure 1 when all applications of $f$ are implemented by $A$. Then $\operatorname{lift}(A)$ denotes while $(A, \operatorname{lifted}(A))$.

Lemma 3 If $A$ in $\mathcal{P}$ has semantics $f$, then lift $(A)$ is in $\mathcal{P}$ and has semantics lift $(f)$.

Of course, we may appeal to Lemma 3 repeatedly to define higher-order versions $\operatorname{lift}(\operatorname{lift}(A))$ and so forth for algorithms $A$ in $\mathcal{P}$ with semantics $f$, which are all in $\mathcal{P}$ by virtue of this lemma. Next, we use these operators for data-driven refinement.

\section{Data-driven refinement}

Let us first consider partial solver

$$
p s_{1}=\text { while }(s c c, p p, \text { fa, ari,gfa) }
$$

Based on the semantics of while $\left(f_{1}, \ldots, f_{k}\right)$, we may assume that the input domain of each $f_{j}$ with $j>1$ equals $\bigcap_{i=1}^{j-1} \Sigma_{f_{i}}$. In particular, if some partial solver $f_{j}$ requires that its input games have no fatal attractors, this is guaranteed by having $f_{l}=f a$ for some $l<j$. We will also that a new analysis $f_{k+1}$ (which may be more expensive, say) is only ever called in the refinement while $\left(f_{1}, \ldots, f_{k+1}\right)$ on states that are residual for while $\left(f_{1}, \ldots, f_{k}\right)$.

Some static analyses below will merge a set of nodes $X$ to a sole node owned by player $p$ and of color $d$. This merge operation can be defined generically:

Definition 2 Let $s$ be a state, $X \subseteq V^{\prime}$ with $|X| \geq 2$ and $X \bullet E^{\prime} \backslash X \neq \emptyset$. Let $p$ be a player, $d a$ color, and $z \notin s . V^{\prime}$. Then tuple merge $(s, X, p, d, z)$ denotes

$$
\text { (s. } \left.W_{0}, s . W_{1}, \text { merge }(\rho, X, z), \text { merge }\left(s . G^{\prime}, X, p, d, z\right), G\right)
$$



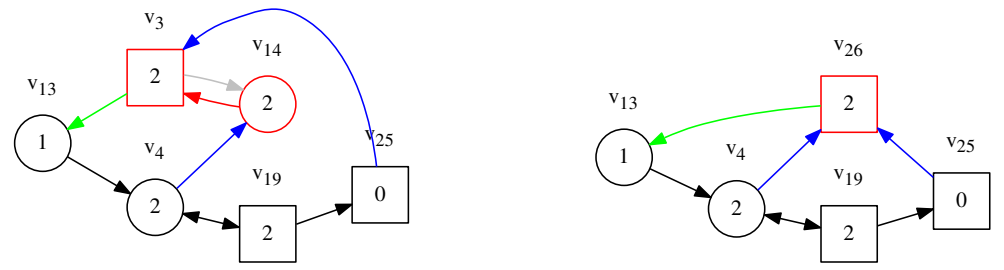

Figure 2: Left: Residual game $s . G^{\prime}$ for $p s_{1}$. Right: game $m_{s s}(s) \cdot G^{\prime}$ obtained from the call merge $\left(s,\left\{v_{14}, v_{3}\right\}\right.$, owner $\left.\left(v_{3}\right), c\left(v_{3}\right), v_{26}\right)$

where the parity game merge $\left(s . G^{\prime}, X, p, d, z\right)$ is defined as $\left(V^{*}, V_{0}^{*}, V_{1}^{*}, E^{*}, c^{*}\right)$ with

$$
\begin{aligned}
V_{1-p}^{*} & =V_{1-p}^{\prime} \backslash X \\
V_{p}^{*} & =\left(V_{p}^{\prime} \backslash X\right) \cup\{z\} \\
E^{*} & =\left(E^{\prime} \backslash X \times X\right) \cup\left(\left(E^{\prime} \bullet X \backslash X\right) \times\{z\}\right) \cup\left(\{z\} \times\left(X \bullet E^{\prime} \backslash X\right)\right)
\end{aligned}
$$

and $c^{*}(v)=c^{\prime}(v)$ for all $v \neq z$ whereas $c^{*}(z)=d$. Relation merge $(\rho, X, z)$ is $\left(\rho \backslash X \times s . V^{\prime}\right) \cup$ $\{(z, w) \mid w \in X \bullet \rho\}$.

Whenever we invoke the above merge method, we need to ensure that the resulting tuple is an actual state. The parity game merge $\left(s . G^{\prime}, X, p, d, z\right)$ has no dead-ends: this is so since $z$ has at least one outgoing edge, which is guaranteed by the fact that $(x, v)$ is in $s . E^{\prime}$ for some $x$ in $X$ and some $v$ in $s . V^{\prime} \backslash X$. Next, we present two static analyses that use this merging.

Sole successor node merging: $m_{s s}$. An inspection of residual games for $p s_{1}$ identifies a method $m_{s s}$ for merging two nodes, so we set

$$
p s_{2}=\text { while }\left(s c c, p p, f a, \text { ari }, g f a, m_{s s}\right)
$$

To see how $m_{s s}$ works, let $s$ be a state in $\Sigma$. Suppose that there are two nodes $v$ and $w$ in $s . G^{\prime}$ such that $v \bullet E^{\prime}=\{w\}, w \bullet E^{\prime} \nsubseteq\{v, w\}$, and the color of $v$ in $s . G^{\prime}$ is not smaller than that of $w$. Choose some $z$ not in the node set of $s . G^{\prime}$. Then $m_{s s}(s)=\operatorname{merge}(s,\{v, w\}$, owner $(w), c(w), z)$. As $w \bullet E^{\prime}$ contains a node not in the merge set $\{v, w\}$, state $m_{s s}(s)$ is well defined. If there are no such nodes $v$ and $w$, we set $m_{s s}(s)=s$. Figure 2 shows a residual game for $p s_{1}$ and the effect of $m_{s s}$ on it: node $v_{14}$ is $v$, node $v_{3}$ is $w$, the owner of $w$ is player $1, w$ has color 2 , and $z$ is $v_{26}$.

Theorem 2 The static analysis $m_{s s}$ is in $\mathcal{P}$.

Merging SCCs: $m_{s c c}$. The study of residual games for $p s_{2}$ introduces more complex methods for merging nodes. We will only describe one of these next, static analysis $m_{s c c}$ which operates on states residual for $f a$ and attempts to merge an SCC in a sub-game of the residual game. For state $s$, this analysis checks whether there is some color $d$ such that the following can be realised: Let $H=\left(V^{\prime}[Z], E^{\prime} \cap Z \times Z\right)$ be the game graph that restricts the game graph of s.G $G^{\prime}$ to $Z=\left\{w \in V_{1-p}^{\prime} \mid c(w) \geq d\right\}$, the set of all nodes $w$ owned by player $1-p$ and of color $\geq d$ in 

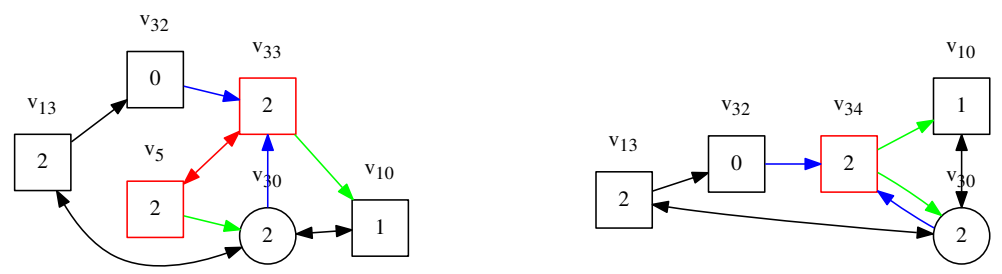

Figure 3: Left: Residual game $s . G^{\prime}$ for $p s_{2}$. Right: game $m_{s c c}(s) . G^{\prime}$ obtained from the call $\operatorname{merge}\left(s,\left\{v_{5}, v_{33}\right\}, 1-0,2, v_{34}\right)$

s. $G^{\prime}$ where $p=d \% 2$. Suppose there is an SCC $C$ in $H$ and a subset $X \subseteq C$ with $|X|>1$ such that all elements in $X$ have color $d$ and where $X \bullet E^{\prime} \cap\left(V^{\prime} \backslash X\right) \neq \emptyset$ in $G^{\prime}$. The latter implies $m_{\text {scc }}(s)=\operatorname{merge}(s, X, 1-p, d, z)$ is well defined: since $X \bullet E^{\prime} \cap\left(V^{\prime} \backslash X\right) \neq \emptyset$, the parity game $m_{s c c}(s) . G^{\prime}$ contains no dead-ends. If there is no such color $d$ with corresponding $H$ and $X$, we set $m_{s c c}(s)$ equal to $s$. This defines a refined partial solver

$$
p s_{3}=\text { while }\left(s c c, p p, f a, \text { ari }, g f a, m_{s s}, m_{s c c}\right)
$$

Figure 3 shows a residual game for $p s_{2}$ and the effect of $m_{s c c}$ on it: $d$ is $2, p$ is 0 , node set $X$ is $\left\{v_{5}, v_{33}\right\}$, and $z$ is $v_{34}$.

The soundness proof for this analysis is pretty straightforward: first we show that the same player indeed wins all nodes in $X$, and then we show that the merged version of the continuation game has the same winning region modulo $\rho$.

Theorem 3 The static analysis $m_{s c c}$ is in $\mathcal{P}$ with domain $\Sigma_{f a}$.

Edge removal based on conditional fatal attractors: $e r_{f a}$. The residual games of $p s_{3}$ led us to studying edge removal methods for states in $\Sigma_{f a}$. We discovered static analysis $e r_{f a}$ which works as follows for any $s$ in $\Sigma_{f a}$ : If there is an edge $(v, w)$ in $s . G^{\prime}$ such that $s . G_{(v, w)}^{\prime}$ has a fatal attractor, then $e r_{f a}$ choses one such edge and sets $e r_{f a}(s)=s \backslash(v, w)$, i.e. $e r_{f a}$ removes edge $(v, w)$ from $s . G^{\prime}$. The intuition is that any fatal attractor that would appear in $s . G_{(v, w)}^{\prime}$ would have to be a fatal attractor for player $1-\operatorname{owner}(v)$, since $s$ is in $\Sigma_{f a}$. Therefore, we may remove the edge $(v, w)$ from $s . G^{\prime}$ as choosing this edge would lead player owner $(v)$ to lose that node. Otherwise, if no such edge exists, $e r_{f a}(s)$ equals $s$. For refined partial solver

$$
p s_{4}=\text { while }\left(s c c, p p, f a, a r i, g f a, m_{s s}, m_{s c c}, e r_{f a}\right)
$$

Figure 4 shows $s . G^{\prime}$ for some $s$ in $\Sigma_{p s_{3}}$ and the effect of $e r_{f a}$ on it: $d$ is $2, p$ is 0 , set $X$ is $\left\{v_{5}, v_{33}\right\}$, and $z$ is $v_{34}$.

Theorem 4 The static analysis er $r_{f a}$ is in $\mathcal{P}$ with domain $\Sigma_{f a}$. 

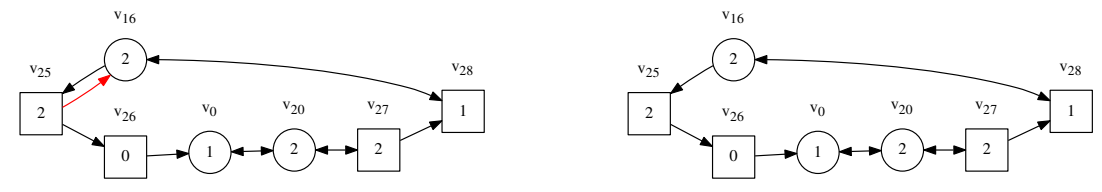

Figure 4: Left: Residual game $s . G^{\prime}$ for $p s_{3}$. Right: game $e r_{f a}(s) \cdot G^{\prime}$ which removes edge $\left(v_{25}, v_{16}\right)$ since $s . G_{\left(v_{25}, v_{16}\right)}^{\prime}$ contains $\left\{v_{25}, v_{16}\right\}$ as a fatal attractor for color 0

Edge removal based on shared descendant: $e r_{s d}$. Residual games for partial solver $p s_{4}$ suggested to us the following static analysis $e r_{s d}$, which removes an edge based on a shared descendant. This checks, for $s$ in $\Sigma$, whether there are three different nodes $v, w, z$ in $s . G^{\prime}$, an edge $(v, w)$ in $s . G^{\prime}, p$ in $\{0,1\}$, and two colours $c_{v}$ and $c_{w}$ in $\mathrm{C}\left(s . G^{\prime}\right)$ (not necessarily at $v$ or $w$ ) with $c_{v} \preceq_{p} c_{w}$ such that:

- there is a path $P_{v z}$ of color $c_{v}$ from node $v$ to $z$ in $s . G^{\prime}$ such that all nodes on $P_{v z}$ are in $V_{p}$ or have only one outgoing edge in $s . G^{\prime}$, and

- there is a path $P_{w z}$ of color $c_{w}$ from node $w$ to $z$ in $s . G^{\prime}$ such that all nodes on $P_{w z}$ are in $V_{1-p}$ or have only one outgoing edge in $s . G^{\prime}$.

If there are such data, $e r_{s d}$ chooses one such edge $(v, w)$ and sets $e r_{s d}(s)=s \backslash(v, w)$, i.e. edge $(v, w)$ is removed from $s . G^{\prime}$. The intuition is that this only requires an argument when player $p$ wins $v$ in $s . G^{\prime}$ with a winning strategy that moves from $v$ to $w$ : then we can employ a dominance argument based on $\preceq_{p}$ as indicated below. Otherwise, if no such edge exists, $e r_{s d}(s)$ equals $s$. Figure 5 shows the effect of $e r_{s d}$ on a residual game for $p s_{4}: v$ is $v_{0}, w$ is $v_{20}, p$ is $1, z$ is $v_{8}$, the path $P_{v z}$ (blue, via $v_{16}$ and $v_{21}$ ) has color $c_{v}=0$, and the path $P_{w z}$ (green, via $v_{19}$ ) has color $c_{w}=0$. This yields a refined partial solver

$$
p s_{5}=\text { while }\left(s c c, p p, f a, a r i, g f a, m_{s s}, m_{s c c}, e r_{f a}, e r_{s d}\right)
$$

The proof of the correctness of $e r_{s d}$ exploits that removing an edge $(v, w)$ where $v$ is in $V_{p}$ cannot increase the winning region of player $p$. Therefore, it will suffice to show that this does not decrease the winning region of player $p$. Only the case when $v$ is won by player $p$ with a strategy that moves from $v$ to $w$ is of real interest: then, any winning strategy for player $p$ at node $v$ in $s . G^{\prime}$ can be replaced with a winning strategy in $s . G^{\prime} \backslash(v, w)$ that moves from node $v$ along the path $P_{v z}$. In detail, we then use this strategy $\tau$ and the path $P_{v z}$ to define a new strategy $\gamma$ with finite memory for that player on the new game $G^{\prime \prime}=s \cdot G^{\prime} \backslash(v, w)$. We then show that this new strategy $\gamma$ is winning in game $G^{\prime \prime}$ on the old winning region of $s . G^{\prime}$, by showing that each infinite play in game $G^{\prime \prime}$ conformant with the new strategy $\gamma$ determines an infinite play in game $s . G^{\prime}$ that is conformant with the (winning) strategy $\tau$, such that the outcome for player $p$ of the infinite play in game $G^{\prime \prime}$ is better or equal with respect to $\preceq_{p}$ to the outcome of the infinite play in game $s . G^{\prime}$. This ensures that player $p$ wins the infinite play in the new game $G^{\prime \prime}$, as he does win the infinite play in game $s . G^{\prime}$.

Theorem 5 The static analysis $\mathrm{er}_{\text {sd }}$ is in $\mathcal{P}$. 


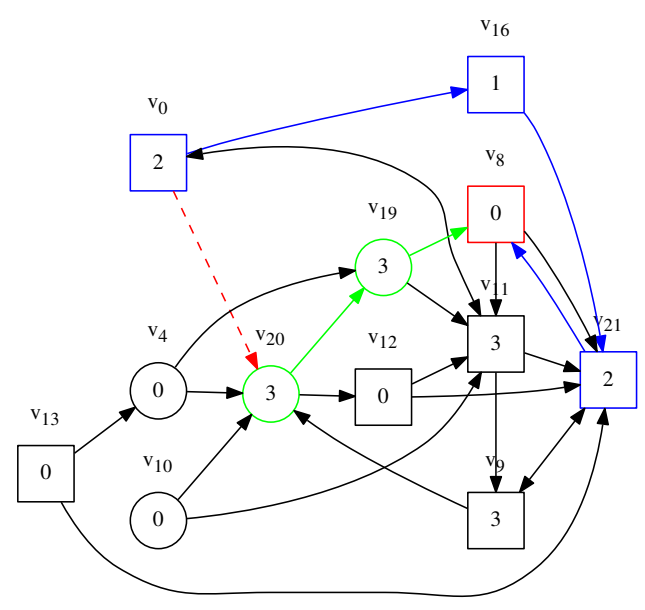

Figure 5: Left: Residual game $s . G^{\prime}$ for $p s_{4}$. Right: game $e r_{s d}(s) \cdot G^{\prime}$ which removes edge $\left(v_{0}, v_{20}\right)$ since it has two control paths that meet the criteria for $e r_{s d}$

\section{Experimental results}

Our approach and its implementation in Python do not compute winning strategies since soundness proofs for some partial solvers require finite memory; related to that, in [12] it was noted that the partial solver psolc, to which while $(g f a)$ in our paper is similar, may require finite memory. We use PGSolver [6, 7] as a test oracle to validate that our implementations of partial solvers are sound, i.e. that they never misclassify the winner of a node of an input game.

Experiments on structured benchmarks. We ran $p s_{1}$ on Keiren's comprehensive benchmark suite [17] on a HP EliteDesk 800 G1 TWR with RAM 16GB and an Intel Core i7-4770 $3.40 \mathrm{GHz}$. For efficiency reasons, we ran $p s_{1}$ over all games in that suite whose textual representation was less than 200KB. This suite contains the PGSolver benchmarks as well; however, for some of the latter types Keiren's suite only contains games whose textual representation is larger than 200KB; for these types we thus used PGSolver itself to generate such test games. We refer to [1] for the full list of these games. In this manner, we tested 481 games - some of which with more than 10,000 nodes. Both $p s_{1}$ and our implementation of Zielonka's algorithm solved 464 of these games completely and agreed on those solutions. For the remaining 17 games, an exception was raised (stack overflow or a timeout of 60 seconds) for at least one of $p s_{1}$ or our implementation of Zielonka's algorithm. Our version of Zielonka's algorithm was also extensively tested against the PGSolver command pgsolver - global recursive, justifying its use in validation testing. That use allowed us to unit test more efficiently, as our pipe from Python to PGSolver input was rather slow.

Random games used. We used a standard type of random game [7] with configuration $x x$ $y y$ - $a a-b b$, which has $x x$ nodes whose ownership is determined uniformly at random, $y y$ colours where colours of nodes are independently and uniformly drawn from set $\{0,1, \ldots, y y\}$, and where 
for each node $v$ the set $v \bullet E$ has at least $a a$ and at most $b b$ elements; the cardinality of $v \bullet E$ is determined for each node independently and uniformly at random.

Unit testing for our implementation of solvers. For each of the four new analyses of Section 4, we generated a stream of random games and applied the analysis to each game as often as it would result in state changes. For each state change, we tested whether the winning regions (modulo potential node merging via $\rho$ ) won't change. Specifically, we generated 100,000 such tests for each analysis. For $e r_{f a}$, we used configuration 60-30-2-3 and 100,395 games in $\Sigma_{f a}$ to generate that many tests. For $e r_{s d}$, we used configuration 60-30-2-3 with 24,081 games, for $m_{s s}$ we took configuration 60-30-1-3 and 100,140 games in $\Sigma_{f a}$, and for $m_{s c c}$ we had configuration 60-30-1-3 with 1,885,423 games. Note that such tests may generate fewer games than test cases, if the analysis can be applied repeatedly on continuation games. But we may have to generate more games than tests, which was the case for analyses that require states from $\Sigma_{f a}$.

In addition, we did unit testing of partial solvers $p s_{1}$ through to $p s_{5}$ : we generated 10 million games of type 50-25-2-4 as a test harness; these partial solvers $p s_{1}$ through to $p s_{5}$ never misclassified a node for all of these games, based on the regression test with PGSolver as described above. Here we also unit tested that these are refinements: $p s_{1} \geq p s_{2} \geq p s_{3} \geq p s_{4} \geq p s_{5}$. This gave us high confidence that these implementations are correct. So we turned unit tests off in further experiments that explored billions of random games in search for residual games.

Finally, we unit tested lifted $(\cdot)$ on 974 residual games that we found for $p s_{5}$ and ran lifted $\left(p s_{5}\right)$ on those: for all of these games this call removed at least one edge (i.e. it reached the if or elseif branch in Figure 1) and it successfully tested that no winning regions changed. We did the same unit tests on 24,132 residual games for $p s_{4}$ that we generated. For each of these games, the if or elseif branch was reached and the resulting game did not change winning regions.

Comparing effectiveness of new analyses. We wanted to understand how often these four analyses can simplify games. For this, we considered states in $\Sigma_{f a}$ to create an input common to all these analyses. We generated 100,000 states $s$ in $\Sigma_{f a}$ where $s . G^{\prime}$ is the result of eliminating all fatal attractors from a random game of configuration type 60-30-2-3. The analyses simplified 99,596 such games for $e r_{f a}, 84,126$ games for $e r_{s d}, 80,327$ for $m_{s s}$, and 7,946 for $m_{s c c}$. Then we did a similar experiment for 25,360 residual games of a partial solver similar to $p s_{3}$, whose residual games are all in $\Sigma_{f a} \cap \Sigma_{e r_{f a}} \cap \Sigma_{m_{s s}}$ : this confirmed that neither $e r_{f a}$ nor $m_{s s}$ simplified any of these games - whereas $e r_{s d}$ simplified 25,355 of these and $m_{s c c}$ simplified 20,119 of these.

Experiments for data-driven refinement. We conducted experiments to determine which random game configurations $x x-y y-a a-b b$ are more prone to generating residual games for our partial solvers above: when $b b$ equals $a a+2$ and $x x$ and $y y$ are fixed, we noticed that $b b=2$ was most effective at generating residual games whereas $a a \geq 5$ was very ineffective. Fixing $a a$ and $b b$ and letting $y y$ be $x x$ or $x x / 2$, we noted that residual games occur more frequently as $x x$ increases from 30 to about 90 but then occur less frequently again. Fixing only yy, we noted that an increase beyond 15 did not have much effect. These insights informed a large experiment in which we generated 10,422, 420 random games of type 50-25-2-3 in total - more than 10 million games - and recorded how many residual games each of the five partial solvers had for these: 32,716 for $p s_{1}, 30,631$ for $p s_{2}, 19,230$ for $p s_{3}, 958$ for $p s_{4}$, and only 136 for $p s_{5}$. 
The latter 136 residual games are completely solved by lift $\left(p s_{5}\right)$. This illustrates that each of the newly discovered partial solvers leads to more effective refinements of existing ones.

Experiments for lift $(\cdot)$. We $\operatorname{ran} \operatorname{lift}\left(p s_{5}\right)$ on a range of random game configurations to see whether we could find any non-empty residual games. We tested this on games of varying configurations with node sizes ranging from 40 to 1000. All of these games, totalling to 9,353,516,890 (over nine billion games), were solved completely by lift $\left(p s_{5}\right)$; specifically, we first ran $p s_{5}$ on these games and invoked lift $\left(p s_{5}\right)$ on all the non-empty residual games, which were only in the order of thousands. This staging is justified as $p s_{5}$ is part of the interaction within $\operatorname{lift}\left(p s_{5}\right)$.

Experiments on large games. The implementation of our game generators and partial solvers is not optimized. For one, it may take too long to generate random games of one million nodes or more. For another, our partial solvers may not be able to solve such large games in a reasonable period of time, be it for random or structured games. Therefore, the insights reported above, including the effectiveness of our proposed data-driven approach to discovering new partial solvers, are limited in that they refer to parity games of small or medium size. However, we did mean to generate - within these performance constraints - some large games and determine whether $p s_{5}$ would be able to solve them completely. The limited number of games that we managed to generate and test in this manner will only provide anecdotal evidence. But we do report such evidence here for sake of completeness.

We tested $p s_{5}$ against the games from PGSolver's structured benchmark that are used in [6]. We solved the Elevator verification problem $G_{n}$ for $2 \leq n \leq 5$ and its variant $G_{n}^{\prime}$ for $3 \leq n \leq 6$. We also solved the Tower of Hanoi problem $T_{n}$ with $5 \leq n \leq 11$. The size of the corresponding games varied from 564 nodes to 708,587 nodes. The partial solver $p s_{5}$ completely solved all of these structured games.

We also tested $p s_{5}$ on large games from the remaining categories of Keiren's comprehensive benchmark suite, namely mlsolver, equivchecking and modelchecking. We managed to generate 15 games across these three types of benchmarks, where the size of these games varied from 35,234 nodes to 1,081, 474 nodes. Partial solver $p s_{5}$ completely solved all of these 15 games.

Based on the experiments on random games that were performed in [6], we finally ran both $p s_{5}$ and lifted $\left(p s_{5}\right)$ on random games with node sets of size either ten thousand, one-hundred thousand, or one million. Specifically, we managed to generate 35, 699 games with 10,000 nodes (32,054 games in configuration 10000-100-2-4 and 3645 games in configuration 10000-1000-2-4), 882 games with 100,000 nodes of configuration 100000-100-2-4, and 17 games with 1,000,000 nodes with configuration 1000000-yy-2-4 where yy equals 100 for six games, 10 for one game, 22 for four games, and 50 for six games. All of these 36,598 games were completely solved by $p s_{5}$, and so therefore also by lifted $\left(p s_{5}\right)$.

\section{Related work}

In [6], a pattern is proposed, implemented, and evaluated for how to solve parity games. This generic solver can be seen as a composition context of partial solvers (in our setting and terminology) in which all but one partial solver run in polynomial time, and where the latter is a complete solver that is only called when the partial solvers cannot progress on any terminal SCC

of the parity game. The aim of this is to gain efficiency, and this was successfully demonstrated 
in [6]. But the aim of our work here is to gain effectiveness so that a composition context of partial solvers would never or very rarely have to call a complete solver. In [8, it is shown that a variant of Zielonka's algorithm solves some classes of parity games in polynomial time, and an improved lower (exponential) bound is derived for solving all parity games with such recursive algorithms. In [13, a function related to lift $(f)$ is studied; using our terminology, it operates as follows: for $w \neq w^{\prime}$ in $v \bullet E$, if there is some node $z$ in $G$ such that $f$ detects a different winner for node $z$ in the two games $G_{(v, w)}$ and $G_{\left(v, w^{\prime}\right)}$, then node $v$ is won by player owner $(v)$ in parity game $G$. It would be of interest to integrate this method into our approach for experimental evaluation. In [11, another function similar to $\operatorname{lift}(f)$ is investigated: apart from presentational differences (our work here uses states), the function in [11] essentially omits the if part of code in Figure 1 and its soundness proof had severe restrictions on the types of partial solvers that it may use as arguments. In [12], experiments compared the effectiveness of partial solver psolB of [10] (which is similar to our while $(f a)$ ) and psolC (which is similar to our while $(g f a)$ ): on random games, psolC was more effective than psolB on games with higher edge density, but not at all more effective on games with lower edge density.

\section{Discussion}

We also ran detailed experiments on residual games of some of the partial solvers $p s_{1}$ to $p s_{5}$. Specifically, we studied structural features of their terminal SCCs. It appears that such SCCs have statistically significant structure. For example, we were unable to find a terminal SCC of a residual game that has two winners; however, we could then manually combine two such games to construct a residual terminal SCC in which both players win nodes.

We implemented the partial solver $e r_{s d}$ in a weaker version than that presented above: control paths only have nodes owned by the controlling player. It may be possible to generalise the $e r_{s d}$ specified in the paper such that node $z$ is reached in the alternating sense by the controlling player (on a tree rather than on a path), and always reached with the specified color.

Our approach to data-driven refinement of partial solvers worked well since residual games were found within a reasonable amount of time. But this method led to powerful partial solvers for which we now genuinely struggle to find any residual games by relying on standard random and non-random benchmarks. This may make it harder to evaluate and improve such a partial solver. Theorem 1, however, suggests one form of evaluation: to prove mathematical properties of residual games that may also imply that well known types of games are never residual for a given partial solver. Partial solver $p s_{1}$, e.g., completely solves all Büchi games, as psolB in [10] does that.

Our paper focussed on effectiveness: the ability of a partial solver to completely solve a game in polynomial time. Our approach can also facilitate the study of the efficiency of composed partial solvers, for example by choosing the order of arguments in while $(\cdot)$.

\section{Conclusions}

There are many heuristics for solving or preprocessing parity games, potentially decreasing the complexity of a parity game by reducing some of its colours, by removing some of its edges, or by removing some of its nodes (whose winners would then be known). Such methods are sound as they do not alter the winning regions of the resulting parity game. We developed here an 
approach to composition that allows such methods to interact and to share information so that their power of inference could be amplified. Concretely, we developed the notion of state that captures computational state within a composition context and defined partial solvers as certain state transformers. Two composition operators for partial solvers were developed and shown to preserve polynomial-time computability: a sequential iteration of a list of partial solvers that tracks progress, and a lift operator testing soundness of edge removals by exploring consequences of edge commitments for a partial solver.

We instantiated these composition operators with partial solvers from the literature and applied them experimentally to study games that such composed partial solvers cannot simplify. These games, seen as data, led to the incremental design of new partial solvers, even to a new method that merges nodes known to have the same but unknown winner. We proved the soundness of these new solvers. Our focus was on computing winning regions, not winning strategies. Would could compute finite-memory winning strategies in principle; it would be interesting to learn whether this could be done here for memoryless winning strategies as well.

We unit tested the implementation of our approach to validate experimental results. The latter demonstrated the effectiveness of such a sequence of refined partial solvers: after only a few refinement steps we arrived at a partial solver that not only solved all structured games from the state-of-the-art benchmark suite for parity games, but whose lifted version also solved all random games generated within a month of calendar time. We think this is compelling evidence that there are very effective polynomial-time partial solvers for parity games.

The strength of this work is that is yields effective partial solvers that are guaranteed to run in polynomial time. But this is also its weakness in that we do not, at present, have a good understanding of what types of parity games are solved completely for certain partial solvers. More powerful versions of Theorem 1, which extend to classes of 2-player games, would be a first step in addressing that weakness.

\section{Open Access to Research Data}

Our source code is openly accessible at

$$
\text { bitbucket.org/Ah-Fat/gandalf_source }
$$

Structured benchmarks we used were not our own and are accessible through the references provided in this paper. We chose not to store the random games we generated. This is justified by the fact that the random generators are publicly available and so these experiments can be

repeated in principle on freshly generated random input, where the expectation is that results will be similar in quality.

Acknowledgements: We thank Nir Piterman very much for his comments on this work.

\section{References}

[1] Ah-Fat, P., Huth, M.: Effective partial solvers for parity games. Technical report 2016/1. Department of Computing, Imperial College London, 33pp., ISSN 1469-4174.

[2] Berwanger, D., Dawar, A., Hunter, P., Kreutzer, S.: DAG-width and parity games. In: STACS 2006, Proceedings of the 23rd Symposium on Theoretical Aspects of Computer Science. LNCS, vol. 3884, pp. 524-436. Springer-Verlag (2006). DOI: doi:10.1007/11672142_43 
[3] Berwanger, D., Grädel, E., Kaiser, L., Rabinovich, R.: Entanglement and the complexity of directed graphs. Theor. Comput. Sci. 463, 2-25 (2012). DOI: doi:10.1016/j.tcs.2012.07.010

[4] Emerson, E., Jutla, C.: Tree automata, $\mu$-calculus and determinacy. In: Proc. 32nd IEEE Symp. on Foundations of Computer Science. pp. 368-377 (1991). DOI: doi:10.1109/SFCS.1991.185392

[5] Fearnley, J., Schewe, S.: Time and space results for parity games with bounded treewidth. Logical Methods in Computer Science 9(2) (2013). DOI: doi:10.2168/LMCS-9(2:6)2013

[6] Friedmann, O., Lange, M.: Solving parity games in practice. In: Liu, Z., Ravn, A. (eds.) Proc. of Automated Technology for Verification and Analysis. Lecture Notes in Computer Science, vol. 5799, pp. 182-196. Springer (2009). DOI: doi:10.1007/978-3-642-04761-9_15

[7] Friedmann, O., Lange, M.: The PGSolver Collection of Parity Game Solvers. Tech. Rep., Institut für Informatik, LMU Munich (Feb 2010), version 3

[8] Friedmann, O.: Recursive algorithm for parity games requires exponential time. In: RAIRO - Theor. Inf. and Applic. 45(4): 449-457, 2011. DOI: doi 10.1051/ita/2011124

[9] Huth, M., Kuo, J., Piterman, N.: The Rabin index of parity games: Its complexity and approximation. Inf. Comput. 245: 36-53 (2015). DOI: doi:10.1016/j.ic.2015.06.005

[10] Huth, M., Kuo, J.H., Piterman, N.: Fatal attractors in parity games. In: Foundations of Software Science and Computation Structures - 16th International Conference, FOSSACS 2013, Lecture Notes in Computer Science, vol. 7794, pp. 34-49. Springer (2013). DOI: doi 10.1007/978-3-642-37075-5_3

[11] Huth, M., Kuo, J.H., Piterman, N.: Fatal attractors in parity games: Building blocks for partial solvers. CoRR abs/1405.0386 (2014)

[12] Huth, M., Kuo, J.H., Piterman, N.: Static analysis of parity games: alternating reachability under parity. In: Semantics, Logics, and Calculi - Essays Dedicated to Hanne Riis Nielson and Flemming Nielson on the Occasion of Their 60th Birthday, January 2016, Copenhagen, Denmark. Lecture Notes in Computer Science, vol. 9560, pp. 159-177. Springer (2016). DOI: doi:10.1007/978-3-319-27810-0_8

[13] Huth, M., Piterman, N., Wang, H.: A workbench for preprocessor design and evaluation: toward benchmarks for parity games. ECEASST 23 (2009). DOI: doi:10.14279/tuj.eceasst.23.324

[14] Jurdziński, M.: Small progress measures for solving parity games. In: Proc. 17th Symp. on Theoretical Aspects of Computer Science. Lecture Notes in Computer Science, vol. 1770, pp. 290-301. Springer-Verlag (2000). DOI: doi:10.1007/3-540-46541-3_24

[15] Jurdziński, M., Paterson, M., Zwick, U.: A deterministic subexponential algorithm for solving parity games. In: Proceedings of ACM-SIAM Symposium on Discrete Algorithms. pp. 117-123. ACM/SIAM (2006)

[16] Jurdziński, M.: Deciding the winner in parity games is in UP $\cap$ co-UP. Inf. Process. Lett. 68, 119-124 (November 1998). DOI: doi:10.1016/S0020-0190(98)00150-1

[17] Keiren, J.J.: Benchmarks for parity games. In: Proc. of Int'l Conf. on Fundamentals of Software Engineering (FSEN). Springer (2015). DOI: doi:10.1007/978-3-319-24644-4_9

[18] Mostowski, A.W.: Games with forbidden positions. Tech. Rep. 78, University of Gdańsk (1991)

[19] Schewe, S.: An optimal strategy improvement algorithm for solving parity and payoff games. In: Kaminski, M., Martini, S. (eds.) Computer Science Logic, 22nd International Workshop, CSL 2008, 17th Annual Conference of the EACSL, Bertinoro, Italy, September 16-19, 2008. Proceedings. Lecture Notes in Computer Science, vol. 5213, pp. 369-384. Springer (2008). DOI: doi:10.1007/978-3-54087531-4_27

[20] Vöge, J., Jurdziński, M.: A discrete strategy improvement algorithm for solving parity games. In: Proc 12th Int. Conf. on Computer Aided Verification. Lecture Notes in Computer Science, vol. 1855, pp. 202-215. Springer (2000). DOI: doi:10.1007/10722167_18

[21] Zielonka, W.: Infinite games on finitely coloured graphs with applications to automata on infinite trees. Theoretical Computer Science 200(1-2), 135-183 (1998). DOI: doi:10.1016/S03043975(98)00009-7 